\title{
The influence of temperature on optical properties of merocyanine dye thin films
}

\author{
M.M. Sieryk ${ }^{1}$, T.P. Doroshenko ${ }^{2}$, K.P. Grytsenko ${ }^{2}$, O.M. Navozenko ${ }^{1}$, O.I. Tolmachev ${ }^{3}$, \\ Yu.L. Slominski ${ }^{3}$, S. Schrader ${ }^{4}$ \\ ${ }^{I}$ Taras Shevchenko Kyiv National University, \\ ${ }^{2}$ V. Lashkaryov Institute of Semiconductor Physics, NAS of Ukraine \\ ${ }^{3}$ Institute of Organic Chemistry, NAS of Ukraine, \\ ${ }^{4}$ University of Applied Sciences Wildau, Germany \\ Corresponding authorse-mail: serikmykola@gmail.com,Tompavl54@gmail.com,d.grytsenko@gmail.com
}

\begin{abstract}
Merocyanine dye thin films have been investigated using optical microscopy methods. The films were deposited using the method of thermal evaporation. The influence of substrate type and temperature on optical properties and spatial orientation of molecules in the above films has been studied. The influence of annealing on structural reorientation of molecules in thermally deposited dye films has been determined.
\end{abstract}

Keywords: merocyanine dyes, thin film, vacuum deposition, optical spectroscopy.

Manuscript received 23.10.12; revised version received 18.12.12; accepted for publication 26.01.13; published online 28.02.13.

\section{Introduction}

The progress in the development of nanotechnology requires to create new photosensitive materials. Recently, more attention has been paid to organic compounds, among them are polymethine (cyanine) dyes due to their photophysical and photochemical properties in the visible and near infrared range of the spectrum [1]. They are used as spectral sensitizers in manufacturing technology of photographic materials [2] and laser components [3]. Nanofilms of polymethine dyes are widely used in: (i) recording media of writeonce compact discs [4], (ii) photovoltaic [5-8], (iii) photosynthetic systems [9], (iv) medicine as components of medical microprobes, microcapsules, etc. [10].

Most of applications require thin films of dyes. Methods for obtaining thin films of organic compounds and polymethine films, in particular, are as follows: Langmuir-Blodgett [11], spin-coating [12], sol-gel [13], etc. However, aggregates formation, influence of external factors on film morphology and optical properties are not well studied. In most of these films, preparation methods involve dye solutions, from which the films are obtained after some processing. It complicates the preparation process of a pure dye due to requirements of the solvent purity, type and concentration.

We have used the method of thermal evaporation. This method was successfully applied to producing nanoscale phthalocyanine films [14], squaraine [15]. The advantages of this method are homogeneity and uniformity of the thickness in the deposited films; lack of solvents in the manufacturing process ("dry" process); ability to prepare multi-component and multi-layered systems [16] in a single technological cycle; control of the film thickness during the deposition process, substrate temperature control in a wide range, direct determination of optical characteristics in the process of dye deposition.

Formation of aggregates, the influence of external factors on optical properties are not sufficiently studied up to date. Thus, our aim was to investigate the influence of substrate type and temperature on optical properties of dye thin films. To determine optical properties of cyanine films at high temperature, the annealing of dye films was performed with simultaneous registration of the transition spectrum. 


\section{Experimental}

A high value of the absorption coefficient in the visible and near infrared regions is one of the main requirements for using dyes in optoelectronic devices. The intensity of absorption is inversely proportional to the bandwidth. That's why the dyes with narrow absorption bands should be used. Merocyanines, as a subclass of polymethine dyes, fulfill this requirement and are technologically suitable for vacuum deposition [18].

Merocyanine dyes were selected to prepare thin films absorbing in the visible region of the spectrum. These dyes are high-polarized $\pi$-electron systems. They are sensitive to the external environmental influence and are able to change the dipole moment substantially in the state of excitation and sensibilize different physicochemical processes [17]. In our investigation, we use merocyanine dyes, formulas of which are presented in Fig. 1.

All the investigated dyes have the same acceptor group with tret-butyl group as a substituent (for stabilization of molecules). The donor group is different for each of the dyes. Pyridine-quinuline exchange in the dye 7477 leads to extension of the $\pi$-electron system of donor fragment and increase of the dipole moment of dye molecule. The donor fragments of the dyes 7496 and 7497 are modified by exocyclic phenylic and phenyl groups, respectively. As a result, it influences on $\pi$ electron system more tangibly and leads to increase of the molecule dipole moment.<smiles></smiles>

7477

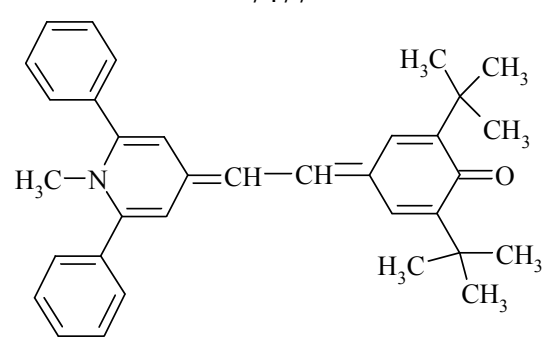

7496

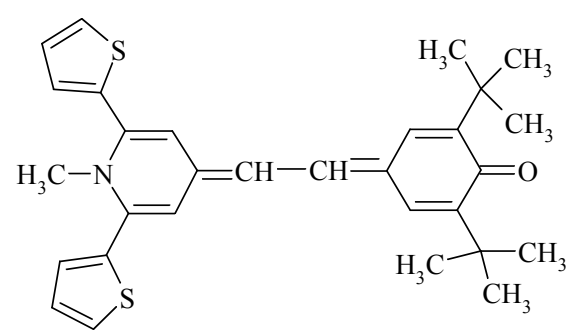

7497

Fig. 1. Chemical formulas of the investigated polymetine dyes.
Structure optimization in the program package Gaussian 03 for an individual molecule in vacuum for each investigated merocyanine dye was done (Fig. 2). The central part of all the molecules (the main chromophore) is flat with the torsional angle close to $0^{\circ}$. Insertion of substituents complicates molecule spacing construction. External substituents are taken out from the plane of the main chromophore due to the presence of methyl group near the nitrogen atom. The calculation shows that in the individual molecule of the dye 7477, quinuline lies in the plane of the main chromophore, but in molecules of the dye 7496, phenyl groups are turned on-the-miter near the angle $90^{\circ}$ to the plane of central part. Phenyl groups in the dye 7497 are set on-the-miter near the angle $54^{\circ}$ to the plane of the main chromophore.

The improved thermal vacuum method was applied to obtain organic thin films. Using this method allows to avoid molecule destruction in the moment of their evaporation. The unit for obtaining organic thin films is designed on the basis of the universal vacuum post VUP-5M. The dyes were deposited at the pressure $10^{-3} \mathrm{~Pa}$ using the special developed evaporator with a silica crucible that was heated resistively. The deposition rate was controlled by changing the heating power and was measured by the frequency change of a quartz resonator. The deposition rate was within the range $0.05 \ldots 0.1 \mathrm{~nm} / \mathrm{s}$.

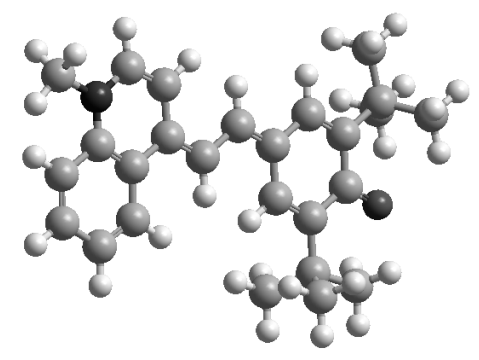

7477
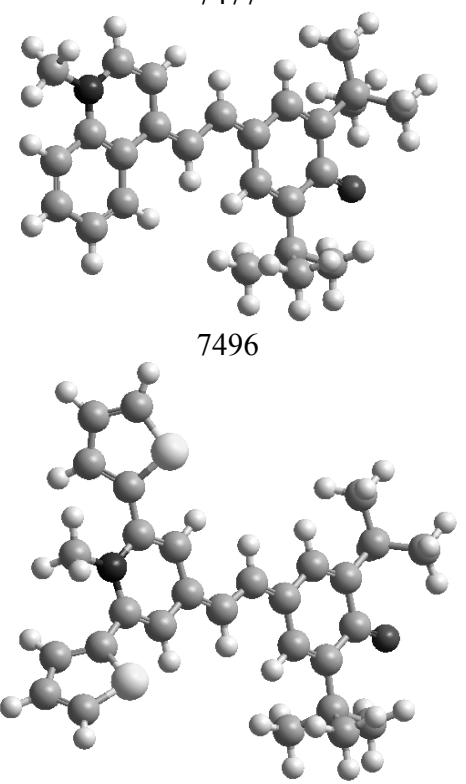

7497

Fig. 2. Optimized 3d-structures of merocyanines.

(C) 2013, V. Lashkaryov Institute of Semiconductor Physics, National Academy of Sciences of Ukraine 
The films were precipitated on two substrates glass ones and those covered with a thin film of PTFE (polytetrafluorethylene) - that were heated in the oven to the temperature $70^{\circ} \mathrm{C}$, and two other identical substrates supported in holders without heating. Special cassettes were used for that. Thicknesses of films were: $170 \mathrm{~nm}-$ for PTFE, $40 \ldots 60 \mathrm{~nm}$ - for dye. Optical transmission spectra of these thin films were measured by the spectrometer ETA-SD30 in the visible range of radiation spectrum.

\section{Results and discussion}

Our measurements of the absorption spectra for synthesized dyes in dichloromethane solution showed that intensive absorption of the obtained dyes takes place within the range $600 \ldots 650 \mathrm{~nm}$.

Individual molecular spectra are typical for merocyanines with characteristic narrow high-intensive absorption bands. Considerable changes in their spectral characteristics were observed under transfer of the solutions to solid films. Moreover, the nature of these changes was different depending on the substrate temperature. The absorption spectra of dye thin films and dye absorption maximum in dichloromethane solution are shown in Fig. 3. In solid films of the investigated dyes, creation of two wide absorption bands occurs. One of these bands is shifted gypsochromely with reference to the monomer band position and the other one is shifted multichromely. It's known [18] that both absorption bands correspond to the first electron transition. The absorption band that corresponds to the second electron transition is located in a more shortwave region, and its intensity is less than monomeric one by several orders.

It is noted that the substrate type doesn't influence on the film absorption peak position, but the absorption intensity difference is 10 to $15 \%$ and even higher for different dyes. Summarized in Table are the values of absorption spectra peaks for the investigated dyes in thin films as compared with $\mathrm{CH}_{2} \mathrm{Cl}_{2}$ solution.

Splitting, large shifting and spectral absorption band broadening in thin film in comparison with solution occur as a result of interaction between dye molecules [19]. Dye association proceeds on the substrate surface during deposition; it is not excluded, however, that dye molecules can also evaporate in the form of associates but not as individual molecules. As it is known [20], dye molecule interaction in film leads to appearance of various aggregates, for example dimers. Due to molecule interaction, the splitting of the first electron level occurs complying with the Davidov rules. In dimers, as a rule, two absorption bands are created. One of them is shifted to the long-wave region, the other one - to the shortwave one relatively to that of the monomer. The spectrum may be complicated during formation of more composite aggregates.

The ratio of intensities for split bands depends on the angle $\alpha$ (see Fig. 4) that is formed by molecules in dimer. When parallel or antiparallel arrangement of molecules takes place in dimer, the intensity of the hypsochromic band grows and intensity of the bathochromic band tends to zero. When the angle is $180^{\circ}$, the intensity, inversely, concentrates in the longwave region, but not in the short-wave one, which is forbidden. For the other angles, there are intermediate values. The intensity ratio for absorption bands caused by dimer molecules oriented at the angle $\alpha$ can be expressed by the following equality:

$\frac{I_{\mathrm{I}}}{I_{\mathrm{II}}}=\operatorname{tg}^{2} \alpha$,

where $I_{\mathrm{I}}$ is the maximum intensity of the hypsochromic absorption band, $I_{\mathrm{II}}-$ maximum intensity of the bathochromic absorption band, $\alpha$ - angle between the molecules in a dimer (see Fig. 4).
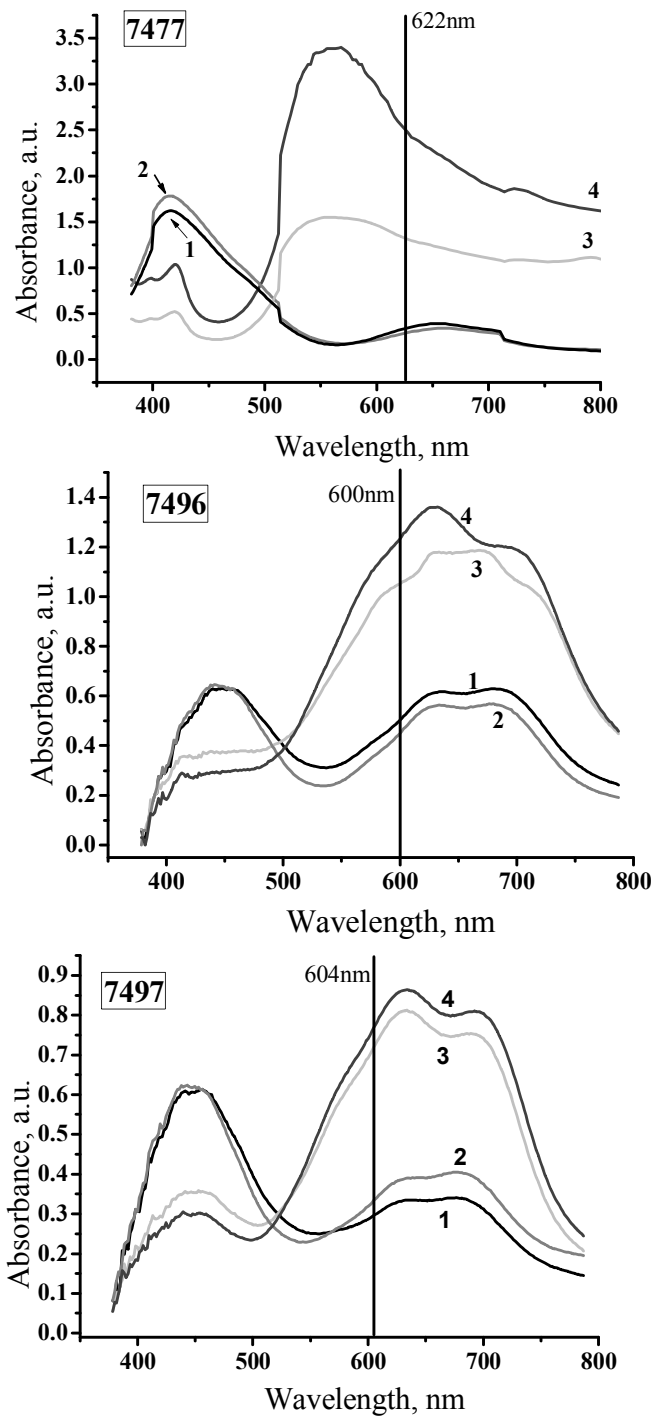

Fig. 3. Absorption spectra of polymethine dye thin films under various conditions of their deposition on: PTFE substrate at room temperature (1), glass substrate at room temperature (1), PTFE substrate at the temperature $70{ }^{\circ} \mathrm{C}(3)$, glass substrate at the room temperature $70{ }^{\circ} \mathrm{C}(4)$.

\section{(C) 2013, V. Lashkaryov Institute of Semiconductor Physics, National Academy of Sciences of Ukraine}


Table 1. Absorption spectra maxima of merocyanines in solution and solid nanofilms obtained by vacuum evaporation, where $n$ means the dye film precipitated on the substrate heated up to $70{ }^{\circ} \mathrm{C}$.

\begin{tabular}{|c|c|c|c|}
\hline Dye & $\begin{array}{c}\text { Absorption } \\
\text { peak position } \\
\text { in } \mathrm{CH}_{2} \mathrm{Cl}_{2} \\
\text { solution (nm) }\end{array}$ & $\begin{array}{c}\text { Absorption } \\
\text { peak position } \\
\text { in film (nm) }\end{array}$ & $\begin{array}{c}\text { Spectral shift } \\
(\mathrm{nm})\end{array}$ \\
\hline 7477 & 622 & 415,655 & $-207,33$ \\
\hline $7477 \mathrm{n}$ & 622 & 420,560 & $-202,-62$ \\
\hline 7496 & 600 & 444,660 & $-156,60$ \\
\hline $7496 \mathrm{n}$ & 600 & 435,632 & $-165,32$ \\
\hline 7497 & 604 & 454,663 & $-150,59$ \\
\hline $7497 \mathrm{n}$ & 604 & 451,632 & $-153,28$ \\
\hline
\end{tabular}

Table 2. Calculated spacing angles $\alpha$ of dimers and the intensity ratio for the absorption bands $I_{I} / I_{I I}$ in the spectra of merocyanine thin films.

\begin{tabular}{|c|c|c|}
\hline Dye dimer & $\begin{array}{c}\text { Angle } \alpha, \\
\text { degrees }\end{array}$ & $\begin{array}{c}\text { Ratio of intensities } \\
I_{\mathrm{I}} / I_{\mathrm{II}}, \text { r.u. }\end{array}$ \\
\hline 7477 & 21.2 & 4.2 \\
\hline 7496 & 43.8 & 1.0 \\
\hline 7497 & 37.5 & 1.8 \\
\hline
\end{tabular}

The distance between these absorption bands is defined by the distance $L$ between molecule centers in the aggregate. When $L$ increases, the distance between the bands also increases and vice versa.

As shown above, it follows from the film spectra that in thin films of the dyes 7477, 7497, the hypsochromic absorption band is more intensive than the bathochromic one. However, in the film of the dye 7496, they are almost equal. The distance between bands changes insignificantly from $210 \mathrm{~nm}$ for the dye 7497 to $240 \mathrm{~nm}$ for the dye 7477. So, one can suggest that in the given films the distance between centers of molecules in dimer is almost equal, and the angle $\alpha$ is the least in dimers of the dyes 7477 and 7497 .

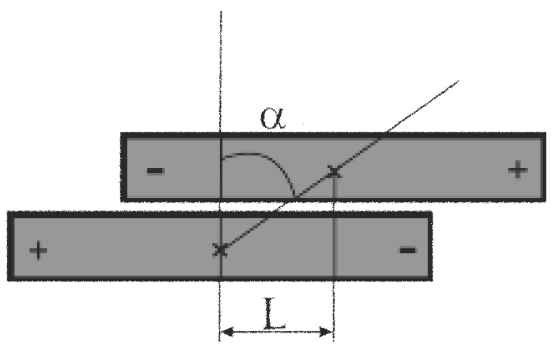

Fig. 4. Model of molecule mutual position in the dimer.

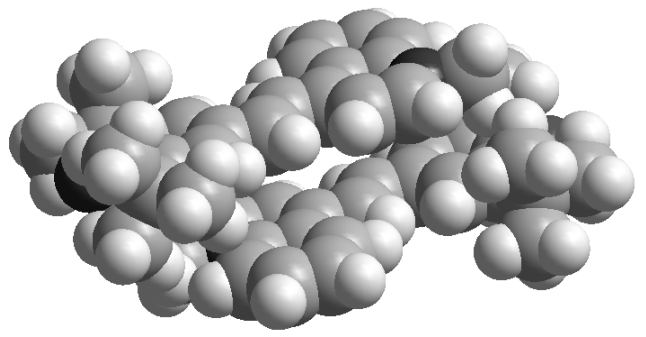

a

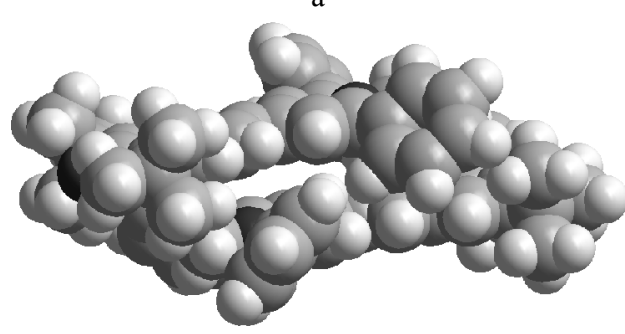

b

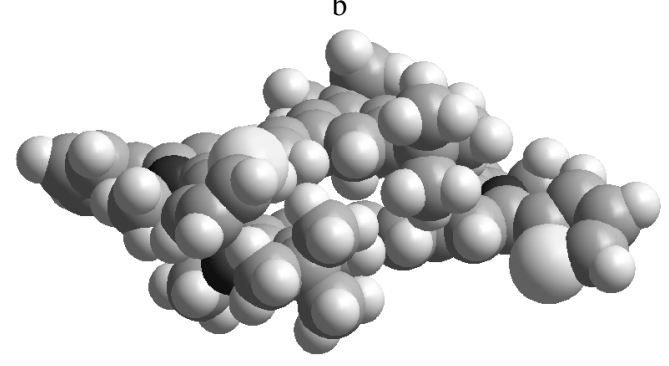

c

Fig. 5. Profiles of investigated merocyanines in the dimer: dye 7477 (a), dye $7496(b)$, dye 7497 (c).

To confirm these assumptions, models of dimers for all the merocyanine dyes were constructed and calculated. Their profiles are shown in Fig. 5. When designing and calculating the dimer model, it was taken into account that dye molecules were arranged in a crystal beyond $3.4 \AA$ between planes of the main chromophores [18]. When constructing these models, the spacing molecule structure and direction of molecule dipoles were also taken into consideration. We suppose that due to strong van der Waals molecular interaction in dimer, molecules begin to change their spacing structure. Final groups try to dispose in the plane of the main chromophore.

The angle $\alpha$ and intensity ratio $I_{\mathrm{I}} / I_{\mathrm{II}}$ were calculated for our dimer models. The results are given in Table 2 . One may see that the calculated angles confirm the main influence of dimer molecules on spectral characteristics of the merocyanine thin films.

At higher temperatures of substrate, the intensity of the hypsochromic absorption band of dyes 7496, 7497 films approaches to zero, as a result, huge broadening the bathochromic band and its intensity rise occur. We suppose that at such substrate temperature, chaotic arrangement and reconstruction of dimers happen. Dye molecules in dimer shift stronger one against another, which by-turn leads to the angle $\alpha$ arise and, respectively, to a significant increase of the absorption 


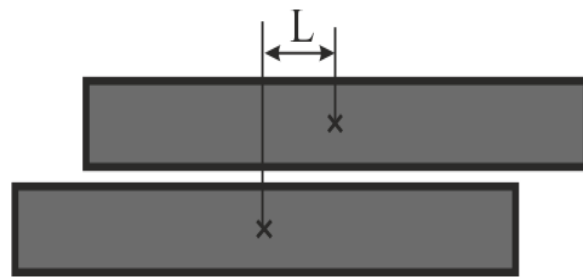

$20^{\circ} \mathrm{C}$

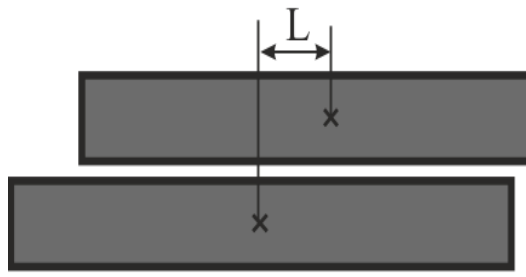

$20^{\circ} \mathrm{C}$

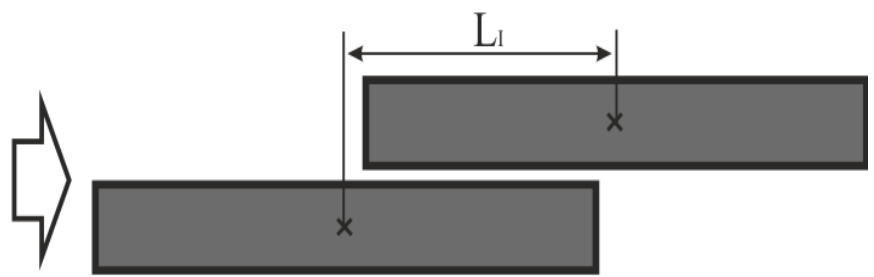

$70^{\circ} \mathrm{C}$

a

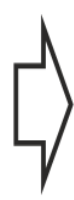

$\mathrm{b}$

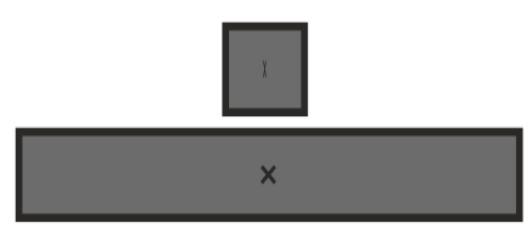

$70^{\circ} \mathrm{C}$

Fig. 6. Models of reorientation of dye molecules in dimer at higher temperatures: for dyes 7496, $7497(a) ; 7477(b)$.

intensity in the long-wave region (Fig. 6). Chaotic arrangement causes significant broadening the absorption band. In the spectrum of the dye 7477 thin film (Fig. 3, curves 3 and 4), the high-intensive hypsochromic absorption band appears in the region near the monomer band. Other bands are absent. In this case, we suppose that higher temperature assists orthogonal arrangement of dye molecules in dimer. Thus, splitting becomes minimal, and the maximum of absorption approaches to that of an individual molecule [20].

To confirm the assumption about reorientation of the dye 7477 molecule in dimer, we annealed in air the film of merocyanine that was precipitated on the substrate at room temperature (Fig. 7).

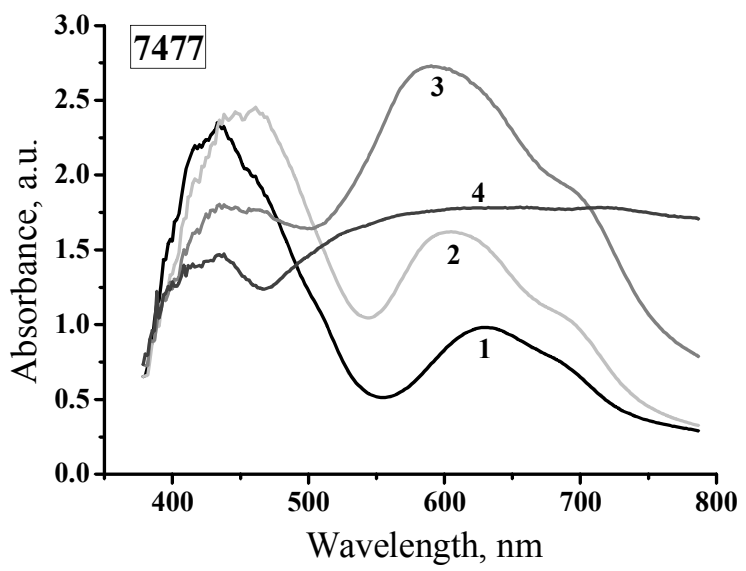

Fig. 7. Change of the dynamics of absorption spectra in the process of annealing of the dye 7477 film precipitated on the substrate at room and elevated temperatures: $20{ }^{\circ} \mathrm{C}(1), 100(2)$, $130(3), 160(4)$.
It's noted that at higher temperatures the hypsochromic peak levels, and instead of it the bathochromic peak shifts to the short-wave region. The molecules of the merocyanine 7477 film in dimer on heating don't shift one against another, but turn and take orthogonal orientation. The shape of spectrum becomes similar to the film spectrum precipitated on the substrate at high temperatures. On further heating, strong broadening the absorption region is observed. It is accompanied by molecule disorder in the film.

\section{Conclusions}

The results of investigations concerning the aggregation processes in dye films, influence of the substrate type, and that of temperature and annealing regimes on optical characteristics of dye thin films have been presented in this paper:

1. Molecule aggregation in thin film with dimer creation is typical for investigated merocyanines. Calculations using the dimer dye models correlate with our analysis of the film absorption spectra.

2. Composition and molecule structure influence on molecule arrangement in the dimer, as it is seen from their spectral characteristics.

3. The substrate type does not influence on absorption peak position but influences on their intensity and broadening of the absorption bands.

4. Substrate temperature influences on orientation of molecules in dimer. It is seen from the absorption spectra: the intensity increases and the absorption band expands in the range of 500 to $750 \mathrm{~nm}$, the intensity decreases within the range $400 \ldots 500 \mathrm{~nm}$.

5. The annealing of films leads to reconstruction and changing the dimer structure orientation in these 
films. Displacement or rotation of the molecule occurs with respect to neighboring molecules in the dimer. High temperatures lead to disordering the molecules. The weak frequency dependence of film absorption in the optical range after annealing at $160{ }^{\circ} \mathrm{C}$ confirms this assumption.

\section{References}

1. A.A. Ishchenko, Structure and Spectralluminescent Properties of Polymethine Dyes. Kyiv, Naukova dumka, 1991, pp. 8-12, 189-206 (in Russian).

2. S.F. Mason, Color and the electronic state of organic molecules. In: K. Venkataraman, Editor, The Chemistry of Synthetic Dyes. Academic Press, New York, 1974, V.3, p. 1708-1709.

3. P. Czerney, G. Granesz, E. Birckner, F. Vollmer, W. Rettig, Molecular engineering of cyanine-type fluorescent and laser dyes // J. Photochemistry and Photobiology A: Chemistry, 89(1), p. 31-36 (1995).

4. V. Shelkovnikov, A.I. Plekhanov, N.A. Orlova, Nanometer films of polymethine dyes in optical memory and nonlinear optics // Nanotechnology in Russia, 3(9-10), p. 35-57 (2008).

5. F.A. Castro et al., On the use of cyanine dyes as low-bandgap materials in bulk heterojunction photovoltaic devices // Synthetic Metals, 156, p. 973-978 (2006).

6. B. Fan et al., Enhanced cyanine solar cell performance upon oxygen doping // Organic Electronics, 9, p. 85-94 (2008).

7. B. Fan et al. High performing doped cyanine bilayer solar cell // Organic Electronics, 11, p. 583588 (2010).

8. V.Y. Merritt, Organic photovoltaic materials: Squarylium and cyanine-TCNQ dyes // J. Res. Develop. 22(4), p. 353-371 (1978).

9. Z. Dai, L. Dähne, E. Donath, and H. Möhwald, Mimicking photosynthetic two-step energy transfer in cyanine triads // Langmuir, 18(12), p. 45534555(2002).

10. L. Dahne, C.S. Peyratout, Tailor-made polyelectrolyte microcapsules: From multilayers to smart containers // Angew. Chem. Int. Ed. 43, p. 3762-3783(2004).

11. L.M. Blinov, Langmuir films // Uspekhi Fiz. Nauk, 155(3), p. 443-480 (1988), in Russian.
12. L. Dahne, Self-organization of polymethine dyes in thin solid layers // J. Am. Chem. Soc. 117, p. $12855-12860$ (1995).

13. O.N. Bezkrovnaya, I.M. Pritula, Luminescent properties of rhodamine $6 \mathrm{~g}$ dye in silica sol-gel matrices // Functional Materials, 17(4), p. 433-438 (2010).

14. H. Xi, Z. Wei, Z. Duan, W. Xu, and D. Zhu, Facile method for fabrication of nanostructured $\mathrm{CuPC}$ thin films to enhance photocurrent generation // J. Phys. Chem. C, 112, p. 19934-19938 (2008).

15. H. Bottcher, T. Fritz, John D. Wright, Fabrication of evaporated dye films and their application // J. Mater. Chem. 3(12), p. 1187-1197(1993).

16. G. Koller, S. Berkebile, J. Krenn, F. Netzer, M. Oehzelt, T. Haber, Heteroepitaxy of organicorganic nanostructures // Nano Lett. 6(6), p. 12071212(2006).

17. A.A. Ishchenko, Yu.L. Slominsky, A.I. Tolmachov, Modern achievements in the field of polymethine dyes of A.I. Kiprianov's school // Zhurnal Org. Pharm. Khimii, 7(3), p. 3-24 (2009), in Ukrainian.

18. A.V. Kulinich, A.A. Ishchenko, Merocyanine dyes: Synthesis, structure, properties and applications // Russian Chem. Rev. 78(2), p. 141-164 (2009).

19. G. Behera, P. Behera, Cyanine dyes: Selfaggregation and behaviour in surfactants (A Review) // J. Surf. Sci. Technol. 23(1-2), p. 1-31 (2007).

20. B. Shapiro, Molecular assemblies of polymethine dyes // Russian Chem. Rev. 75(5), p. 484-510 (2006).

21. K. Grytsenko, T. Doroshenko, Yu. Kolomzarov, O. Lytvyn, M. Serik, O. Tolmachev, Yu. Slominski, S. Schrader, Growth and optical properties of dye films and dye-in-polymer matrix deposited by vacuum evaporation // Semiconductor Physics, Quantum Electronics \& Optoelectronics 13(2), p. 177-179 (2010).

22. K. Grytsenko, T. Doroshenko, Yu. Kolomzarov, D. Prescher et al., Research on the growth of dye film in vacuum in situ // Proc. SPIE, 6999, p. 6991z-16991 z-6 (2008).

23. K. Grytsenko, Yu. Kolomzarov, O. Lytvyn, V. Prokopets, O. Tolmachev et al., Nonconventional properties of dye clusters in polytetrafluoroethylene matrix // Abstr. Book Conf. "Physics and Technol. of Thin Films and Nanosystems," May 25-29, 2009, Ivano-Frankivsk, Ukraine, p. 163-164 (2009). 